\title{
Elicitação de requisitos para promover a comunicação de crianças com deficiência intelectual
}

\section{Elicitation of requirements to promote communication of children with intellectual disabilities}

\author{
Tiago Anunciação Ricaldi ${ }^{1}$ and Carla Diacui Medeiros Berkenbrock ${ }^{(\bullet, 1}$ \\ ${ }^{1}$ Universidade do Estado de Santa Catarina \\ *tiagoricaldi@hotmail.com; carla.berkenbrock@udesc.br
}

Recebido: 25/03/2020. Revisado: 04/03/2021. Aceito: 05/04/2021.

\begin{abstract}
Resumo
A comunicação é essencial para o estabelecimento de relações sociais entre os seres humanos. A comunicação pode usar recursos de tecnologia da informação por meio de comunicação aumentativa e alternativa para fornecer a inclusão social de pessoas com deficiência intelectual. Esta pesquisa apresenta a elicitação de requisitos para promover a comunicação e inclusão social de crianças com deficiência intelectual por meio da evolução de um sistema de comunicação aumentativo e alternativo. A metodologia é baseada no processo de engenharia de software do Agile Unified Process e no processo HCI de design centrado no usuário. Os resultados obtidos incluem os testes de usabilidade da ferramenta, a elicitação dos requisitos e os protótipos não funcionais da evolução da ferramenta. As contribuições da pesquisa consistem na definição de requisitos por meio de bases literárias, entrevistas, testes de usabilidade e construção de protótipos não funcionais da evolução do sistema.
\end{abstract}

Palavras-Chave: Comunicação Aumentativa e Alternativa; Deficiência Intelectual; Inclusão Social; Tecnologia Assistiva.

\begin{abstract}
Communication is essential for the establishment of social relations among human beings. Communication can use information technology resources through augmentative and alternative communication to provide the social inclusion of people with intellectual disabilities. This research presents the elicitation of requirements to promote the communication and social inclusion of children with intellectual disability through the evolution of an augmentative and alternative communication system. The methodology is based on the Agile Unified Process software engineering process and the HCI process of user-centered design. The results obtained include the usability tests of the tool, the elicitation of the requirements and the non-functional prototypes of the evolution of the tool. The research contributions consist of the definition of requirements through literary bases, interviews, usability tests, and the construction of non-functional prototypes of the evolution of the system.
\end{abstract}

Keywords: Augmentative and Alternative Communication; Assistive Technology; Intellectual Disability; Social Inclusion.

\section{Introdução}

A comunicação é um meio para que se estabeleçam as relações sociais entre os seres humanos. A comunicação é 
imprescindível para a socialização e a compreensão entre as pessoas. As pessoas se comunicam por meio de diversas formas como: escrita, verbal, gestual, corporal, código braille, Língua Brasileira de Sinais (LIBRAS), Comunicação Aumentativa e Alternativa (CAA), entre outras.

A comunicação pode utilizar recursos da Tecnologia da informação (TI) para proporcionar a inclusão social. De acordo com Luce and Estabel (2020), as tecnologias da informação aliadas à comunicação propiciam a inclusão social e digital e exercem um papel fundamental como instrumentos de mediação entre os sujeitos em uma sociedade na qual o acesso à informação é considerado um direito de todos. Segundo Marques et al. (2010) o surgimento das tecnologias da informação e comunicação (TICs) aplicadas ao contexto educacional, possibilita a utilização de ferramentas para a inclusão de crianças com déficits de aprendizagem derivados da dificuldade cognitiva.

A comunicação é um elemento de inclusão social para as pessoas com deficiência intelectual (DI), porque por meio dela é possível que essas pessoas expressem seus desejos e suas necessidades. A DI, também conhecida como transtorno do desenvolvimento intelectual, de acordo com o Association et al. (2014), é um transtorno com o seu início no período do desenvolvimento que inclui deficits funcionais, tanto intelectuais quanto adaptativos, nos domínios conceitual, social e prático. Esses deficits incluem, por exemplo, dificuldades de escrita, comunicação, convivência social e de cuidados pessoais que podem afetar a autonomia na realização de tarefas diárias de um indivíduo com DI.

O desenvolvimento de tecnologias assistivas de CAA para crianças com DI possui uma complexidade no desenvolvimento pelo fato de envolver diversos aspectos de comunicação como na identificação de imagens, no reconhecimento de palavras, no processo de formação de palavras ou frases, entre outros. Os aspectos de comunicação variam de acordo com cada pessoa com DI, porque essas pessoas possuem suas próprias características e peculiaridades para se comunicar e expressar suas vontades diárias. Diante desse contexto é possível extrair o problema da pesquisa onde construção de sistemas de informação para promover a comunicação de pessoas com DI é um desafio para cientistas da computação. É um desafio porque cada pessoa com DI possui o seu diagnóstico, e consequentemente o nível de comunicação pode variar de um grau leve, moderado, grave até um grau profundo.

\section{As crianças com DI e CAA}

A pessoa com DI, segundo a Association et al. (2014), caracteriza-se por deficits em capacidades mentais genéricas, como raciocínio, solução de problemas, planejamento, pensamento abstrato, juízo, aprendizagem acadêmica e aprendizagem pela experiência. Esses problemas prejudicam o indivíduo na aquisição da autonomia pessoal à realização de suas atividades. Esses deficits resultam em prejuízos no funcionamento adaptativo, de modo que o indivíduo não consegue atingir padrões de independência pessoal. A autonomia na realização das atividades engloba alguns aspectos como a comunicação, participação social, funcionamento acadêmico ou profissional e independên- cia pessoal em casa ou na comunidade.

A DI pode ser classificada de acordo com níveis, podendo ser leve, moderada, grave e profunda (Association et al., 2014). Nas crianças com DI leve existem dificuldades em aprender habilidades acadêmicas que envolvam leitura, escrita, matemática, tempo ou dinheiro. As crianças com DI moderada possuem dificuldades maiores com um lento progresso na leitura, na escrita, na matemática e na compreensão do tempo e do dinheiro ao longo dos anos escolares (Association et al., 2014).

O desenvolvimento desse projeto é fruto de uma parceria entre a Universidade do Estado de Santa Catarina e o Núcleo de Assistência Integral ao Paciente Especial (NAIPE). O NAIPE é uma instituição vinculada à secretaria da saúde e é referência para todo o país no atendimento a pessoa com DI. Essa pesquisa consiste em apresentar a elicitação de requisitos para promover a comunicação e a inclusão social de crianças com DI por meio da evolução um sistema de informação de CAA. Na DI grave existe pouca compreensão da linguagem escrita ou de conceitos que envolvam números, quantidade, tempo e dinheiro. Na DI profunda as habilidades costumam envolver mais o mundo físico do que os processos simbólicos. Ainda conforme a Association et al. (2014), a DI ocorre no período do desenvolvimento, contudo a identificação da DI pode ocorrer em diversos períodos de crescimento, por exemplo, nos diagnósticos de DI grave atrasos em marcos motores, linguísticos e sociais podem ser identificáveis nos primeiros dois anos de vida entre aqueles com DI. Por outro lado, diagnósticos de DI leve podem não ser identificados até a idade escolar, quando ficam aparentes as dificuldades de aprendizagem acadêmica.

As dificuldades de comunicação das pessoas com DI podem ser suprimidas por meio da utilização de métodos da CAA. O campo da CAA concentra-se em apoiar indivíduos com distúrbios severos de comunicação expressiva. A CAA aborda os aspectos da comunicação suplementar que tem por finalidade pesquisar modos alternativos de comunicação para indivíduos com distúrbios de comunicação. A Associação Americana de Fonoaudiologia (Fossett and Mirenda, 2007) define a CAA como um conjunto de práticas clínicas e educacionais que buscam compensar deficiências temporárias ou permanentes, limitações de atividade e restrições de participação de pessoas com transtornos graves da produção ou compreensão fonoaudiológica, incluindo modos falados e escritos de comunicação. A falta de uma comunicação eficaz, faz com que as pessoas com necessidades complexas de comunicação vivam de maneira limitada para expressar necessidades, desenvolver relacionamentos sociais e trocar informações (Caron and Light, 2016).

A CAA envolve um conjunto de conhecimentos que servem para ajudar os usuários que utilizam a CAA, os seus familiares e seus colaboradores (Thiessen and Beukelman, 2019). Esse conjunto de conhecimentos envolvem ferramentas de intervenção de CAA com uma série de soluções classificadas em low-technology e high-technology. A lowtechnology se refere a um conjunto de ferramentas de comunicação que não utilizam dispositivos eletrônicos como: pranchas de comunicação por símbolos, pranchas de comunicação alfabética, pastas de comunicação por figuras, cartões de comunicação. A high-technology diz respeito a 
utilização de ferramentas computacionais para promover e auxiliar na CAA.

A construção de sistemas computacionais de CAA envolvem algumas avaliações a respeito das pessoas que farão uso dessa ferramenta. Essas avaliações englobam a avaliação da comunicação atual, avaliação da compreensão simbólica, avaliação das competências de alfabetização e avaliação das necessidades culturais e familiares. As avaliações são necessárias para que antes de realizar a construção de um sistema de CAA, seja compreendido o impacto das barreiras de oportunidade no desenvolvimento e competência da comunicação, bem como o desenvolvimento de estratégias para remediar essas barreiras para acomodar as diversas necessidades das pessoas que utilizam a CAA e das suas famílias (Fossett and Mirenda, 2007).

A utilização dos sistemas de CAA tem como intuito estabelecer uma melhora na qualidade de vida das pessoas com distúrbios de comunicação. O incentivo de pessoas envolvidas no processo como, familiares, terapeutas e cuidadores no uso da CAA é importante para que a pessoa com barreiras de comunicação mantenha a motivação na utilização do sistema. As pessoas envolvidas no processo também devem estar aptas para apoiar o usuário na utilização do sistema.

\section{Trabalhos relacionados}

Os trabalhos relacionados têm por intuito identificar os requisitos que são responsáveis por promover a comunicação de pessoas com DI ou outras deficiências com limitações na linguagem verbal por meio de tecnologias assistivas de CAA.

de Oliveira et al. (2013) descrevem um aplicativo Android de CAA, denominado VoxLaPS, destinado a auxiliar no tratamento de pacientes com condições ou limitações na linguagem oral. As funcionalidades da aplicação são: escrever texto por meio de pictogramas com legenda, ler texto, excluir texto, usar telas interativas com botões em forma de placa com imagens associadas aos textos e permitir a navegação entre telas. A metodologia de desenvolvimento do sistema utiliza um processo de desenvolvimento ágil não especificado e a metodologia de avaliação do sistema ocorre por meio de experimentos com 14 especialistas da área de acessibilidade.

El-Seoud et al. (2014) propõem um aplicativo móvel de CAA, na plataforma iOS, que permite a comunicação de pessoas com autismo sem discurso verbal. A funcionalidade do sistema tem como objetivo obter como entrada uma sentença árabe e convertê-la automaticamente em suas imagens e fotos correspondentes para realizar a comunicação. A metodologia de desenvolvimento do sistema ocorre por meio de um processo de engenharia de software e a metodologia de avaliação utiliza testes com crianças com autismo para explicar-lhes histórias simples em árabe.

Huijbregts and Wallace (2015) realizaram um estudo de campo para avaliar o uso de um aplicativo iOS de CAA, denominado TalkingTiles, para pessoas com afasia. Esse aplicativo permite aos usuários a construção de frases tocando em blocos associados com as palavras ou frases curtas, podendo ser customizado conforme as necessidades de cada usuário. A metodologia de desenvolvimento não foi especificada e a metodologia de avaliação ocorreu por meio da observação do uso do sistema com quatro pessoas com afasia e também foram aplicadas entrevistas com os participantes, seus parceiros e seus cuidadores em uma instalação de apoio local.

Guisen et al. (2012) apresentam o design de um sistema web colaborativo de CAA, denominado ECCA, para as pessoas com necessidades complexas de comunicação. O primeiro protótipo tem como funcionalidade a criação de mensagens didáticas com pictogramas utilizando um processador de texto. A metodologia de desenvolvimento do protótipo usa a análise de requisitos e prototipação, e a metodologia de avaliação é planejada com especialistas de campo que podem fornecer seu feedback e ajudar a estabelecer a linha de base para o próximo estágio evolucionário.

Ganz et al. (2013) investigaram a eficácia de um aplicativo de Picture Exchange Communication System (PECS) de CAA, em iOS, em comparação ao PECS tradicional (com um livro de comunicação física) em pessoas com autismo e que possuem necessidades complexas de comunicação. A principal funcionalidade é a comunicação por meio de imagens. A metodologia de desenvolvimento não foi especificada e a metodologia de avaliação ocorre por meio da metodologia Single Subject Design com três participantes, onde dois participantes demonstraram uma preferência pelo aplicativo, enquanto o outro preferiu o livro de comunicação tradicional do PECS.

Wilson et al. (2016) descrevem o desenvolvimento de um aplicativo Android de CAA, denominado Put Yourself in the Picture, para apoiar a comunicação de pessoas com deficiência intelectual. Esse aplicativo possui como funcionalidades tirar selfie e sobrepor à imagem escolhida com a selfie utilizando contorno de imagem para projetar uma imagem de si mesmo, e por último compartilhar a imagem final com seus cuidadores. A metodologia de desenvolvimento utiliza a metodologia do design iterativo ágil reflexivo aliado ao planejamento centrado na pessoa e a metodologia de avaliação ocorre por meio da observação do uso e entrevistas.

Baldassarri et al. (2014) apresentam uma ferramenta de CAA em Android, denominada AraBoard, para as pessoas com necessidades complexas de comunicação. A ferramenta é formada por duas aplicações diferentes: o AraBoard Construtor, para a criação e edição dos blocos de comunicação, e o AraBoard Player, para a visualização das placas construídas. A metodologia de desenvolvimento não foi especificada e a metodologia de avaliação da ferramenta ocorreu via questionário por meio de 295 consultas feitas via web. As respostas foram analisadas e demonstraram a alta aceitação dos usuários.

Os trabalhos relacionados englobam aspectos da CAA direcionados para pessoas que possuem pouca ou nenhuma comunicação verbal. O trabalho de Wilson et al. (2016) foi desenvolvido para apoiar as pessoas com DI. O trabalho de Huijbregts and Wallace (2015) foi desenvolvido para apoiar as pessoas com afasia que possuem distúrbios na comunicação oral. Os trabalhos de El-Seoud et al. (2014) e Ganz et al. (2013) foram desenvolvidos para apoiar as pessoas com autismo, e que possuem distúrbios na comunicação oral. Os trabalhos de de Oliveira et al. (2013), Guisen et al. (2012) e Baldassarri et al. (2014) foram desenvolvidos para apoiar as pessoas com necessidades de comunicação no 
geral como: DI, paralisia cerebral, autismo, etc.

A semelhança dos trabalhos relacionados com a pesquisa apresentada neste trabalho é que todos abordam aspectos relacionados com a CAA por meio de placas de comunicação por imagens e vocalização. A diferença dessa pesquisa é que além da comunicação por imagem e vocalização delas, também será integrado por meio de um sistema de CAA um alfabeto para a digitação e vocalização das palavras para crianças com DI em processo de alfabetização. E além desse alfabeto, também será integrada ao sistema uma agenda de atividades com imagens que podem ser vocalizadas para a orientação temporal das atividades a serem realizadas na semana.

\section{Metodologia}

Nessa pesquisa foram empregados testes de usabilidade e o processo de engenharia de software processo unificado ágil (AUP) em paralelo ao processo Interação HumanoComputador (IHC) de design centrado no usuário. Os testes de usabilidade empregados para avaliar a interface da primeira versão do sistema foram aplicados com o primeiro usuário por meio do protótipo funcional. Durante as experiências de uso, são registrados dados sobre o desempenho dos participantes na realização das tarefas e suas opiniões e sentimentos.

No desenvolvimento preliminar da segunda versão do sistema são utilizados o processo de engenharia de software AUP e o processo de design centrado no usuário. A metodologia ágil incentiva o envolvimento ativo do cliente durante todo o ciclo de vida de desenvolvimento de software. Neste trabalho o envolvimento dos clientes na construção dos artefatos é essencial pelo fato de que é um trabalho destinado a um público específico de pessoas com DI. Essa metodologia ágil relata que as entregas desenvolvidas após cada iteração são fornecidas aos usuários para o uso e consequentemente são realizados os aprimoramentos com base no feedback do cliente. Essa metodologia é utilizada porque além de envolver as pessoas interessadas no processo de desenvolvimento ela busca melhorar os processos a cada iteração com base no feedback das pessoas envolvidas no processo como as pessoas com DI, psicólogos, família, educadores especiais, entre outros.

As camadas do AUP, baseado em Flora and Chande (2014), consistem nos seguintes objetivos: A fase de concepção visa identificar o escopo inicial do projeto, obter a aceitação das partes interessadas no projeto, esboçar uma arquitetura potencial para o sistema. A fase de elaboração consiste em validar a arquitetura do sistema. A fase de construção tem por finalidade desenvolver o sistema de forma iterativa e incremental, e que atenda às necessidades de maior prioridade das partes interessadas do projeto. A fase de transição consiste em validar e implantar o sistema no ambiente de produção.

A escolha do design centrado no usuário foi realizada para que seja possível a construção de um artefato computacional que atenda as exigências dos usuários. Nos processos centrados no usuário o designer deve estudar quem são os usuários do sistema, seus objetivos, suas características físicas, cognitivas e comportamentais.

O processo de IHC de design centrado no usuário, nesse

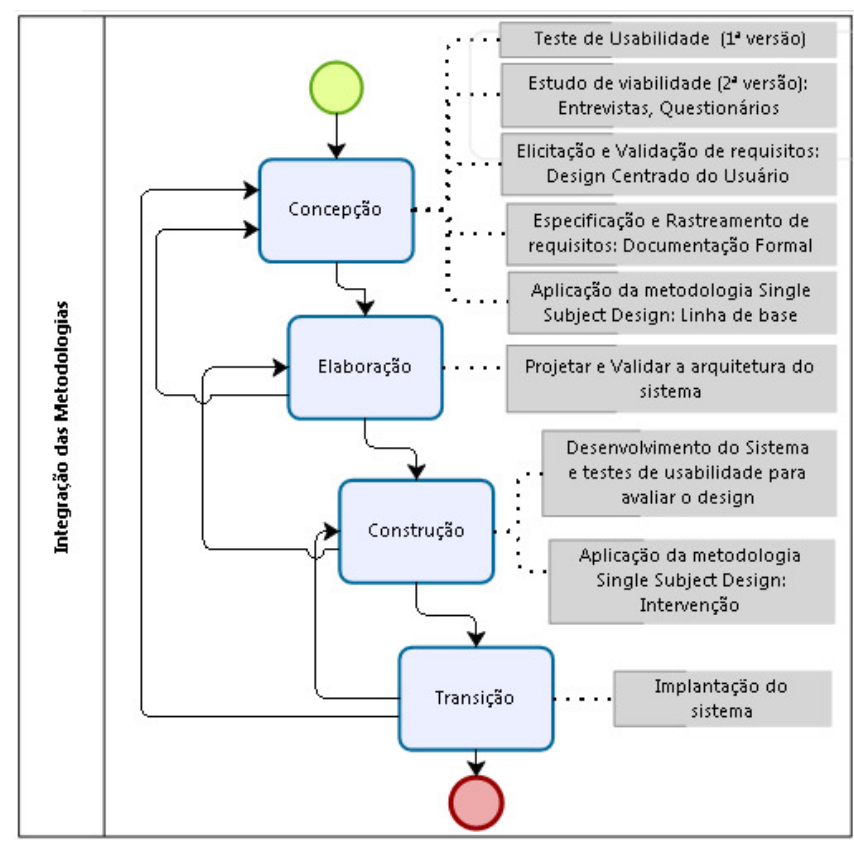

Figura 1: Estrutura da Metodologia

trabalho, é empregado para auxiliar nas seguintes atividades: (i) identificação de requisitos; (ii) validação desses requisitos com a prototipação não funcional; e (iii) validação da versão funcional do sistema junto aos usuários finais e interessados no sistema. Na seção dos resultados estão descritas como essas metodologias estão empregadas no desenvolvimento preliminar dessa pesquisa.

\section{Resultados preliminares}

Os processos que auxiliaram no desenvolvimento preliminar, foram: AUP e o design centrado no usuário. Para coordenar as atividades a serem desempenhadas é necessária a escolha de um workflow para organização metodológica em termos de desenvolvimento do projeto. Na Fig. 1 é possível evidenciar como está planejado o desenvolvimento dessa pesquisa com a integração das metodologias.

Nas subseções referentes à concepção, elaboração, construção e transição mostram as etapas que já foram realizadas no desenvolvimento desse projeto, bem como mencionam as etapas que faltam ser implementadas.

\subsection{Concepção}

A fase de concepção contempla as seguintes atividades: teste de usabilidade, estudo de viabilidade, elicitação e validação de requisitos, especificação e rastreamento de requisitos e, por último a aplicação da linha de base da metodologia single subject design que segue as diretrizes de Horner et al. (2005).

\subsubsection{Teste de usabilidade}

Nessa fase primeiramente foi realizado um teste de usabilidade da primeira versão do sistema para apoiar a comunicação de uma criança com DI leve (Carniel and Berken- 


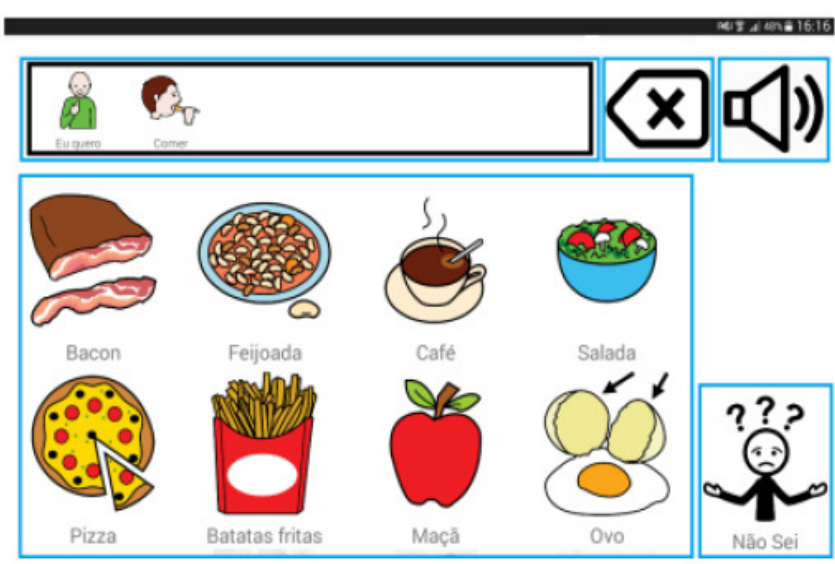

Figura 2: Dispositivo móvel para CAA

brock, 2020). Esse sistema foi desenvolvido para dispositivos móveis utilizando o Sistema Operacional Android. O aplicativo de CAA está representado na Fig. 2.

O sistema de CAA da Fig. 2 estabelece a comunicação por meio de blocos de navegação que vocalizam as imagens. A estrutura dessa navegação segue a estrutura sujeito + ação + opção de ação. As imagens do aplicativo são inseridas por meio de um gerenciador Desktop que são transferidas para o aplicativo.

O teste de usabilidade desse aplicativo foi seguido utilizando as atividades do teste de usabilidade. As atividades são preparação, coleta de dados, interpretação, consolidação dos resultados e relato dos resultados.

Na fase de preparação foi definido que o teste seria realizado com uma criança de 11 anos, denominado usuário $\mathrm{n}^{0} 1$, que possui uma DI considerada leve. Essa criança estava acompanhada da mãe e de uma fonoaudióloga, na instituição de apoio as pessoas com deficiência intelectual, que a auxiliavam quando necessário. Em seguida foram preparadas algumas perguntas com atividades para que o usuário realizasse utilizando o aplicativo de CAA. Essas atividades foram observadas por dois alunos de mestrado e uma professora da Universidade do Estado de Santa Catarina, pela mãe da criança e pela fonoaudióloga. As presenças da mãe e da fonoaudióloga foram importantes porque elas são as pessoas que mais convivem com a criança e seus feedbacks são essenciais para a evolução da pesquisa.

A criança utilizou o sistema de CAA por meio do tablet por cerca de 30 minutos na instituição de apoio as pessoas com deficiência intelectual, e durante as perguntas observamos o seu comportamento utilizando o sistema. Durante a observação foi perguntado à criança qual era o seu nome para que ela respondesse selecionando a sua imagem no sistema de CAA. A criança, por sua vez, procurou as letras do alfabeto para escrever o seu nome, porém o sistema estava sem a funcionalidade para vocalizar o nome completo ao ser digitado, e apenas vocalizava as letras do alfabeto isoladamente.

O usuário quando questionado sobre a comida que mais gostava seguiu o fluxo da navegação do aplicativo "Eu quero + Comer + Batata Frita", porém a pergunta foi apenas o que ela mais gostava, e por meio dessa pergunta foi possível observar que o sistema só permitia uma forma de comunicação para o usuário responder essa pergunta. Com base nisso, observamos que seria necessário adicionar um menu de categorias para dar a possibilidade ao usuário de responder mais objetivamente a questão, sem necessariamente seguir o fluxo "Eu quero + Comer + Batata Frita". No final da sessão do teste de usabilidade observamos que o usuário perdeu o foco na comunicação, pois ele saia do aplicativo para entrar na internet e em aplicativos de jogos. Essa perda de foco do usuário na utilização do sistema fez com que evidenciássemos que o dispositivo (tablet) deveria ser utilizado apenas para a CAA. Após o teste de usabilidade foi realizado um estudo de viabilidade visando o desenvolvimento da segunda versão do sistema.

\subsubsection{Estudo de viabilidade}

O estudo de viabilidade realiza uma estimativa acerca da possibilidade de se satisfazerem as necessidades do usuário identificado e utilizando tecnologias atuais. O estudo de viabilidade consiste em verificar se o produto a ser desenvolvido atende as expectativas dos potenciais usuários. $O$ estudo de viabilidade foi realizado para o desenvolvimento da $2^{\mathrm{a}}$ versão do sistema de CAA para crianças com DI que possuem pouca ou nenhuma comunicação verbal. Esse estudo é realizado por meio de entrevistas informais e questionários com profissionais da instituição de apoio as pessoas com deficiência intelectual. Esses profissionais são: um terapeuta ocupacional, uma fonoaudióloga e uma psicóloga da instituição. Durante o estudo de viabilidade foram realizadas algumas perguntas como: $\left(1^{\mathrm{a}}\right)$ Qual o perfil dos pacientes que você trabalha e a faixa etária? $\left(2^{\mathrm{a}}\right)$ Quais as dificuldades de comunicação enfrentadas pelos pacientes? $\left(3^{\mathrm{a}}\right)$ Você utiliza algum software de CAA para auxiliar nas atividades com os pacientes? $\left(4^{\mathrm{a}}\right)$ Quais as funcionalidades que você acha que um sistema de CAA deve conter para auxiliar nas suas atividades? $\left(5^{\mathrm{a}}\right)$ Que tipo de dispositivo você preferia que fosse utilizado (Tablet, Smartphone, Notebook)? (6 $\left.6^{\mathrm{a}}\right)$ Quais são os pontos positivos e negativos que você considera em um aplicativo de CAA?

As respostas do terapeuta ocupacional foram $\left(1^{\mathrm{a}}\right) \mathrm{DI}$, Síndrome de Down e Transtorno do Espectro Autista. $\left(2^{\mathrm{a}}\right)$ dificuldades na decodificação de informações. $\left(3^{a}\right)$ Não. $\left(4^{\mathrm{a}}\right)$ funcionalidades gerais que deem maior autonomia para o paciente na escola, em casa e na rua. $\left(5^{\mathrm{a}}\right)$ Tablet e smartphone. ( $\left.6^{\mathrm{a}}\right)$ Os pontos positivos são que pode melhorar a comunicação e autonomia do paciente. Os pontos negativos são que o paciente pode perder a vontade de se comunicar e utilize o aparelho para outras coisas como jogar, navegar na internet etc.

As respostas da fonoaudióloga foram. ( $\left.1^{\mathrm{a}}\right)$ DI. $\left(2^{\mathrm{a}}\right)$ sem comunicação verbal e trocas de fala. ( $\left.3^{a}\right)$ Não. $\left(4^{a}\right)$ funcionalidades que atendam as situações de vida no lar, na escola e um aplicativo de texto com leitura global. E também permitir a vocalização em vozes masculina e feminina $\left(5^{\mathrm{a}}\right)$ Tablet e smartphone. ( $\left.6^{\mathrm{a}}\right)$ Os pontos positivos são ampliar ou dar a possibilidade de se comunicar com eficácia. Os pontos negativos são a possibilidade de o paciente não se apropriar adequadamente ao dispositivo.

As respostas da psicóloga foram. ( $\left.1^{\mathrm{a}}\right)$ DI e Síndrome de Down. ( $\left.2^{\mathrm{a}}\right)$ dificuldades na comunicação. ( $\left.3^{\mathrm{a}}\right)$ Já, mas o aplicativo não atendia todas as necessidades do paciente por conter muitas informações na interface, o que causava 


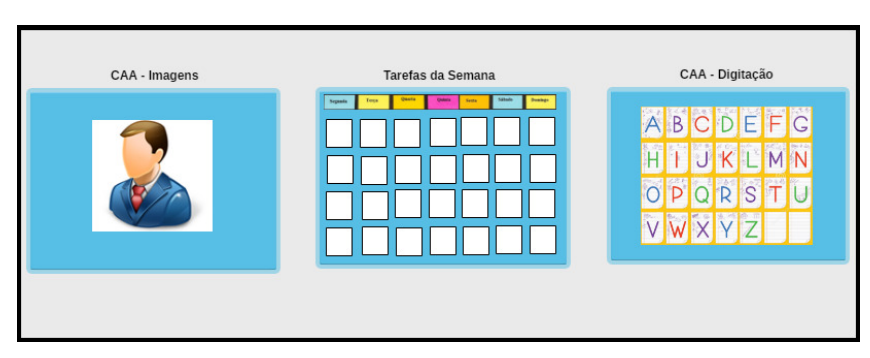

Figura 3: Interface Inicial

confusão na comunicação. $\left(4^{\mathrm{a}}\right)$ Aplicativo de comunicação por imagens, com orientação temporal das atividades diárias do paciente. ( $5^{\mathrm{a}}$ ) Tablet e smartphone. ( $6^{\mathrm{a}}$ ) Os pontos positivos são permitir a comunicação por meio da CAA em dispositivos móveis. Os pontos negativos são que alguns pacientes podem ter restrições na utilização da tecnologia.

Após esse estudo de viabilidade o próximo passo foi realizar a elicitação e a validação dos requisitos com auxílio do processo de IHC de design centrado no usuário.

\subsubsection{Elicitação e validação de recursos}

Primeiramente para realizar a elicitação dos requisitos identificamos as necessidades dos potenciais usuários, para então depois identificar os requisitos. A identificação das necessidades começou com o teste de usabilidade e estudo de viabilidade que já foram relatados.

Neste estágio da pesquisa foi designado um paciente da instituição de apoio às pessoas com deficiência intelectual denominado, usuário $\mathrm{n}^{\mathrm{O}} 2$ com 11 anos de idade, para contribuir com o desenvolvimento da pesquisa. Esse paciente possui síndrome de down e uma deficiência intelectual leve com apraxia na fala e má formação da dentição. Com base na literatura, no teste de usabilidade realizado com 0 usuário $\mathrm{n}^{0} 1$, no estudo de viabilidade desenvolvido com os profissionais da saúde e na entrevista realizada com a mãe e a psicóloga do paciente $\mathrm{n}^{\mathrm{o}} 2$, foi realizado a prototipação não funcional da segunda versão do sistema de CAA descrito nas Fig. 3, 4, 5, 6 e 7 a seguir.

A segunda versão do sistema de CAA está representada na Fig. 3, e contempla três módulos: $\left(1^{\circ}\right)$ A comunicação por imagens e vocalização do som, $\left(2^{\circ}\right)$ Agenda das tarefas a serem realizadas na semana e $\left(3^{\mathrm{a}}\right)$ Digitação das palavras com vocalização.

Na Fig. 4 é possível visualizar o protótipo não funcional da comunicação por imagens. A estrutura da comunicação da $1^{\mathrm{a}}$ versão "Sujeito + Verbo + Opção da ação" está mantida, mas para simplificar e aumentar os modos de comunicação do usuário foi adicionado um menu lateral de categorias. Esse menu permite que o usuário responda alguma pergunta executando somente 2 ações na interface. A interface inicial também foi simplificada em relação à primeira versão. Na primeira versão, por exemplo, a opção "Eu quero" desencadeava em outras subcategorias como "Beber, Comer, etc" que desencadeavam em outras subsubcategorias como "leite, água, etc". Na primeira versão, por exemplo, se o usuário quisesse se comunicar dizendo "Eu quero + Comer + Batata Frita", ele executaria três ações em três telas. Nesta segunda versão, optou-se por deixar os verbos de ação do usuário na interface principal, sendo assim para executar a mesma ação de dizer "Eu quero +

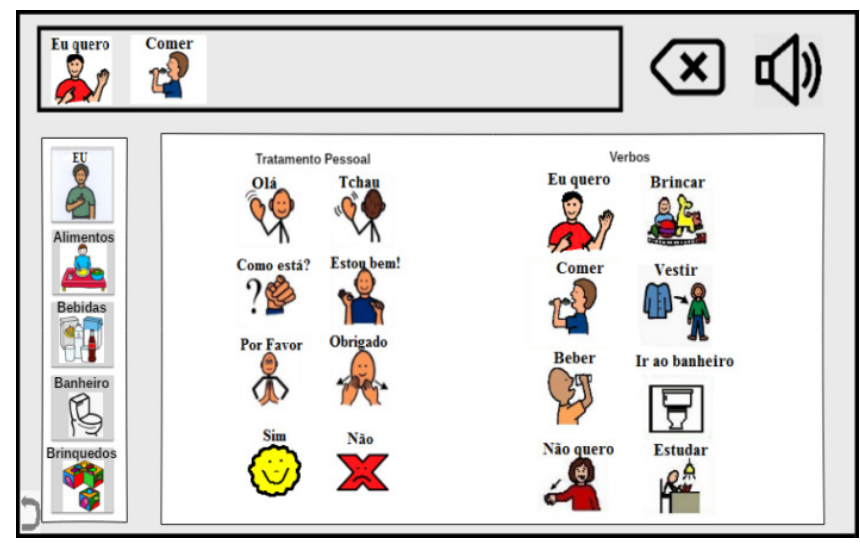

Figura 4: Interface Inicial da CAA por imagens e som

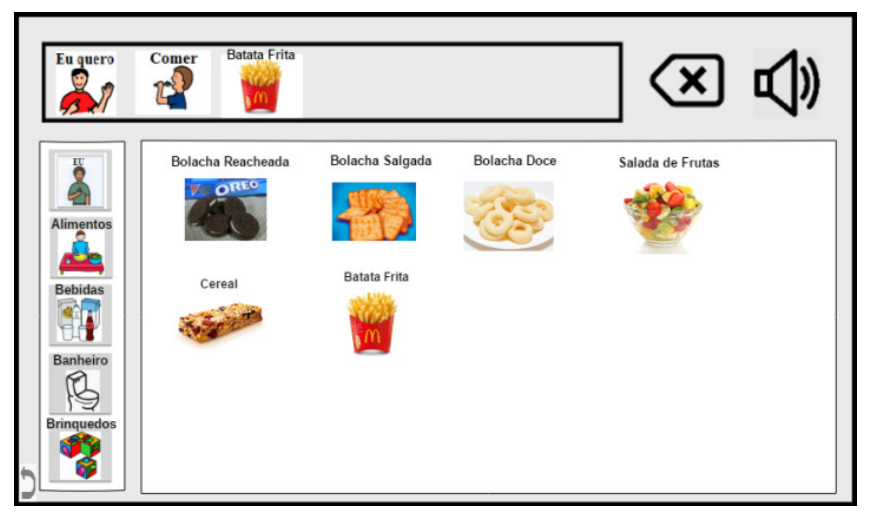

Figura 5: Interface da Comunicação da CAA por imagens e som

Comer + Batata Frita" o usuário executaria a três ações em duas telas, conforme podemos evidenciar nas Fig. 4 e 5.

A Fig. 5 é a sequência da comunicação da Fig. 4 . 0 sistema vocaliza a comunicação do usuário.

Na Fig. 6, está o segundo módulo da prototipação não funcional, da funcionalidade visualizar as tarefas a serem realizadas na semana. A funcionalidade consiste numa grade de tarefas dividida pelos dias da semana em seus turnos da manhã, tarde e noite. Essa funcionalidade permite que os usuários visualizem imagens e legendas sobre cada uma das atividades que eles executam durante a semana. O sistema permitirá a vocalização das atividades para a orientação temporal do usuário.

Na Fig. 7 é possível visualizar o terceiro módulo da prototipação não funcional que é realizar a digitação das palavras e permitir a vocalização do som.

Os protótipos das Fig. 3, 4, 5, 6 e 7 foram mostrados para a psicóloga e a mãe do paciente $\mathrm{n}^{0} 2$ com o objetivo de validar a prototipação, obter seus feedbacks e levantar novos requisitos. A mãe e a psicóloga realizaram essa validação porque são as pessoas que mais convivem com o paciente e também porque elas conhecem os modos de comunicação que mais se adaptam ao paciente. Elas fizeram algumas considerações sobre os protótipos.

No módulo 1 da comunicação por imagens e sons elas relataram os verbos que são mais utilizados pelo paciente 


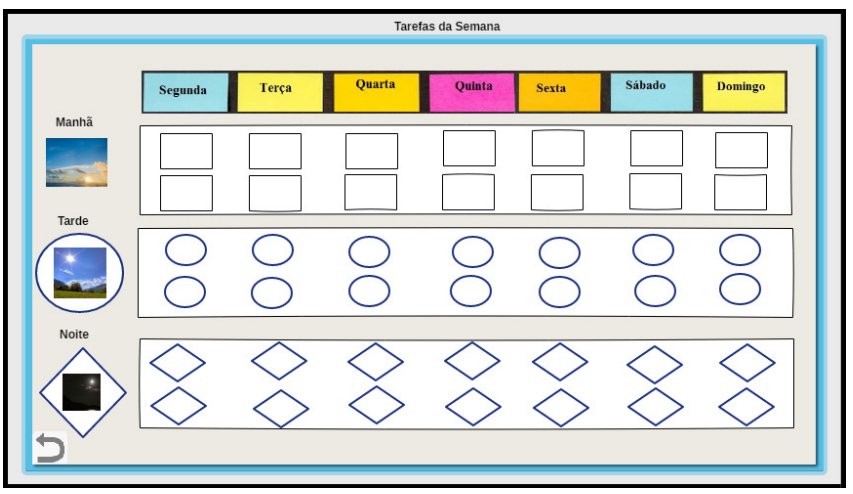

Figura 6: Interface do módulo de adicionar as tarefas a serem realizadas na semana

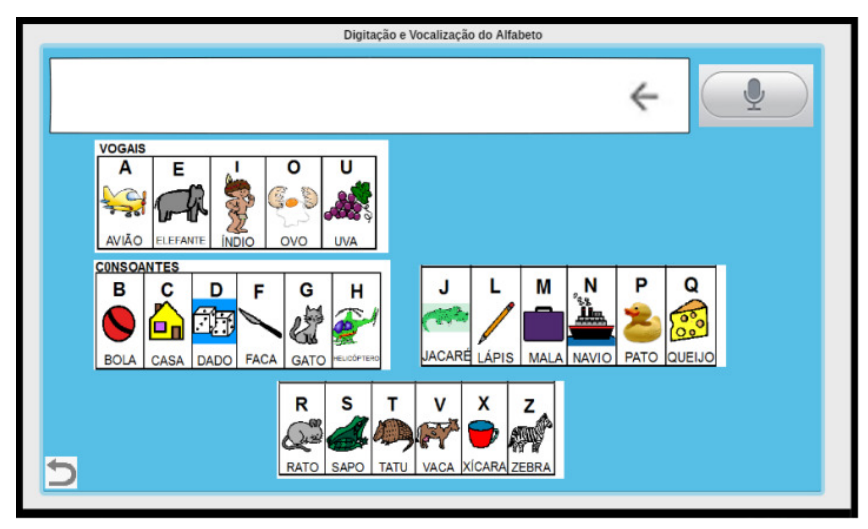

Figura 7: Interface do módulo de realizar a digitação das palavras com vocalização

como: ir, ser, estar e ficar. Também foi relatado que o paciente sabe contar de 1 até 20 e reconhece os números 10,20 , 30, 40 e 50. As imagens da CAA devem ser nítidas, ou seja, apenas a imagem necessária para realizar a comunicação.

No módulo 2, de adicionar as tarefas a serem realizadas na semana foi sugerido que cada linha nos turnos tivesse cores diferentes para auxiliar na identificação da manhã, tarde e noite.

No módulo 3 de realizar a digitação das palavras e obter a vocalização foi sugerido que fosse colocado o alfabeto corrido, sem figuras e sem auto sugestão na escrita das palavras. As próximas etapas do design centrado no usuário de construir uma versão interativa e avaliar essa versão serão realizadas na fase de construção.

\subsubsection{Especificação dos requisitos e rastreamento}

A especificação consiste no detalhamento dos requisitos. A especificação apresenta basicamente uma visão geral do sistema, requisitos específicos e informações complementares. A visão geral em alto nível dos requisitos funcionais do dispositivo de CAA é apresentada nos casos de uso do dispositivo de CAA na Fig. 8.

Os requisitos não funcionais (RNF) serão descritos na Tabela 1.

O rastreamento de requisitos consiste em verificar a relação dos requisitos funcionais e não funcionais conforme

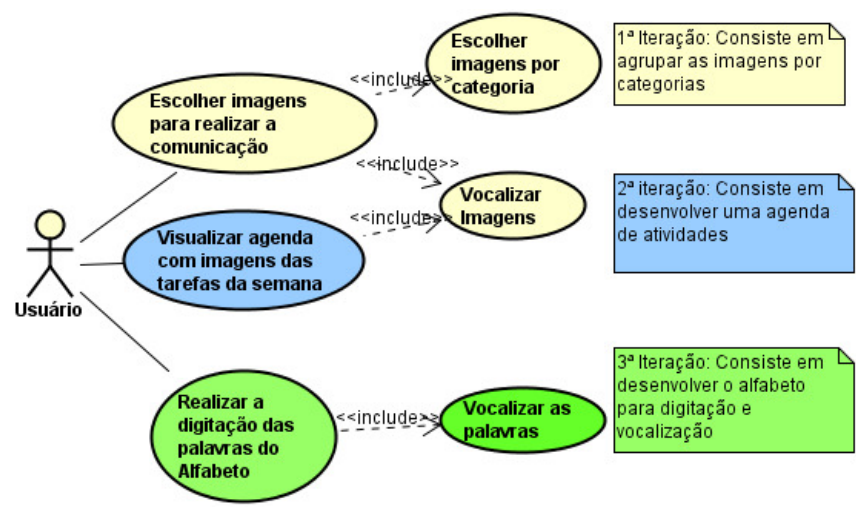

Figura 8: Casos de Uso da CAA do usuário

veremos a seguir. O requisito funcional de escolher as imagens para realizar a comunicação está relacionado com os requisitos não funcionais $\mathrm{RNF} 1, \mathrm{RNF} 2, \mathrm{RNF} 3, \mathrm{RNF} 4, \mathrm{RNF}$ 5 , RNF 6, RNF 7. O requisito funcional de visualizar agenda com imagens e tarefas da semana está relacionado com os requisitos não funcionais $\mathrm{RNF} 1, \mathrm{RNF} 2$, $\mathrm{RNF}$ 3, RNF 4, RNF 5, RNF 7. O requisito funcional de realizar a digitação das palavras do alfabeto e vocalizar está ligado aos requisitos não funcionais RNF 1, RNF 2, RNF3, RNF 7, RNF 8, RNF 9.

\subsubsection{Aplicação da metodologia single subject design}

Conforme Horner et al. (2005), a aplicação dessa metodologia desempenha um papel importante no desenvolvimento de práticas baseadas em evidências no campo da educação especial. Essa metodologia é utilizada no presente trabalho porque ela pode ser empregada para avaliar aspectos a respeito do comportamento e desenvolvimento de pessoas com DI em determinadas atividades. Nessa fase de concepção vamos coletar os dados da eficácia da comunicação do usuário $\mathrm{n}^{0} 2$ para estabelecer uma linha de base sem a intervenção da CAA e da tecnologia. A coleta desses dados foi realizada por meio de um questionário com 3 profissionais da instituição de apoio as crianças com deficiência intelectual. O questionário perguntou qual a eficácia da comunicação do paciente em uma escala likert de 0 a 4, onde 0 quer dizer que não se comunica, 1 quer dizer que se comunica com muita dificuldade, 2 que se comunica parcialmente, 3 que se comunica bem e 4 se comunica muito bem. A comunicação do paciente na visão de todos os profissionais é a escala 2 que significa que ele se comunica parcialmente.

\subsection{Elaboração}

A fase de elaboração consiste na fase de projeto e arquitetura do sistema. A arquitetura do sistema em alto nível consiste em um gerenciador desktop que envia as imagens da CAA, via socket, para o aplicativo de CAA em Android. Essa arquitetura é a mesma utilizada por Carniel and Berkenbrock (2020) na primeira versão do sistema. Na segunda versão do sistema serão adicionadas novas funcionalidades no gerenciador desktop que consistem em adicionar, alterar e excluir agenda de atividades com imagens e legendas, e será adicionado um campo de categorias 
Tabela 1: Requisitos não funcionais do aplicativo de CAA

\begin{tabular}{lc}
\hline RFN & Descrição \\
\hline RNF 1: Sistema & $\begin{array}{c}\text { O tablet deve ser configurado } \\
\text { apenas para a utilização da CAA }\end{array}$ \\
\hline RNF 2: Sistema & $\begin{array}{c}\text { O sistema deve permitir a } \\
\text { vocalização em vozes } \\
\text { masculina e feminina }\end{array}$ \\
\hline RNF 3: Usabilidade & O usuário deve realizar a CAA \\
& navegando no máximo por 2 Telas \\
\hline RNF 4: Usabilidade & $\begin{array}{c}\text { O usuário deve possuir mais de } \\
\text { uma opção para a exploração } \\
\text { a das imagens da CAA na interface }\end{array}$ \\
\hline RNF 5: Usabilidade & Usar fonte simples na legenda \\
& das imagens para não \\
& haver distorções \\
\hline RNF 6: Interface & A interface da CAA do módulo \\
& 1 deve permitir no máximo 16 \\
& imagens para não poluir a interface \\
\hline RNF 7: Desempenho & $\begin{array}{c}\text { Ao clicar numa ação ou imagem } \\
\text { o aplicativo não pode demorar } \\
\text { mais que 2 segundos para executar }\end{array}$ \\
\hline RNF 8: Usabilidade & O alfabeto deve conter somente \\
& letras sem imagens associadas \\
\hline RNF 9: Usabilidade & O campo de digitação do \\
& alfabeto não deve ter a opção \\
& de auto-sugestões \\
\hline
\end{tabular}

para separar as imagens em categorias. Nessa fase ainda serão desenvolvidos futuramente o modelo de domínio da aplicação, e os diagramas de sequência para representar as interações entre os objetos que se comunicam.

\subsection{Construção}

A fase de construção que também será desenvolvida futuramente e se refere a implementação das iterações projetadas e também diz respeito à aplicação dos testes que serão empregados como o teste de usabilidade baseado no design centrado no usuário. Essa fase também contempla a aplicação da fase de intervenção da metodologia single subject design (Horner et al., 2005) com o auxílio da tecnologia. Após a intervenção será realizada a comparação com a linha de base que foi realizada na fase de concepção.

\subsection{Transição}

A fase de transição é referente ao final da fase de testes quando o sistema será validado e implantado no ambiente de produção.

Primeiramente para realizar a elicitação dos requisitos identificamos as necessidades dos potenciais usuários, para então depois identificar os requisitos. A identificação das necessidades começou com o teste de usabilidade e estudo de viabilidade que já foram relatados.

\section{Considerações finais}

A comunicação Aumentativa e Alternativa (CAA) reúne métodos e tecnologias desenhadas para auxiliar ou substituir a comunicação oral de pessoas com limitação de fala. Este trabalho apresenta a elicitação de requisitos para realizar a prototipação da evolução de uma ferramenta de CAA. A efetuação do teste de usabilidade da primeira versão do sistema foi importante para que fossem verificadas algumas melhorias a serem realizadas para a segunda versão no sistema. O estudo de viabilidade com os profissionais da saúde por meio de entrevistas informais e questionários evidenciou a viabilidade na evolução do sistema de CAA e a utilidade do sistema para promover a comunicação de crianças com DI. A prototipação não funcional da segunda versão que foi realizada permitiu que obtivéssemos feedbacks para que possamos aprimorar a interface da segunda versão do aplicativo de CAA em prol dos usuários.

A integração da metodologia ágil de engenharia de software com a metodologia de processo de IHC de design centrado no usuário foi útil porque a metodologia de engenharia de software permite organizar o projeto em etapas que permitem a inserção de novos requisitos ao longo do processo de desenvolvimento. $O$ design centrado no usuário também tem suas etapas bem organizadas, mas tem seu foco voltado mais para o usuário.

O desenvolvimento dessa pesquisa encontrou as algumas limitações. O usuário $\mathrm{n}^{0} 1$ apresentou estado de ansiedade em contato dispositivos móveis. Por esse motivo seus psicólogos acharam melhor interromper as interações por meio do dispositivo móvel de CAA com ele. Essa pesquisa também tinha um terceiro usuário com o qual tivemos um contato preliminar, mas ele teve que ingressar em outra instituição de apoio as pessoas com deficiência e isso fez com que perdêssemos o contato com esse paciente.

Os trabalhos futuros dessa pesquisa consistem em continuar e aprimorar o desenvolvimento das fases de concepção, elaboração, construção e transição. Pretende-se realizar com o paciente $\mathrm{n}^{0} 2$ algumas sessões, para que ele possa escolher algumas imagens manualmente por meio de cartões. Essas sessões são para ele escolher as imagens que melhor se adaptem a comunicação do seu cotidiano, para que, por fim possamos colocar essas imagens na $2^{\mathrm{a}}$ versão do sistema de CAA. Além disso, atualmente esta pesquisa conta com a participação de 3 novos pacientes diagnosticados com DI e que não possuem fala funcional. Os pacientes e seus familiares já estão colaborando para o aperfeiçoamento da ferramenta.

O engajamento de pessoas como os profissionais da saúde, familiares na elicitação dos requisitos é importante para que possamos compreender algumas das necessidades de comunicação de pessoas com DI. Além disso, é necessário que essas pessoas que convivem rotineiramente com os pacientes estejam aptas para motivar e auxiliar o usuário com DI no uso do sistema de CAA.

\section{Referências}

Association, A. P. et al. (2014). DSM-5: Manual diagnóstico e estatístico de transtornos mentais, Artmed Editora.

Baldassarri, S., Rubio, J. M., Azpiroz, M. G. and Cerezo, 
E. (2014). Araboard: A multiplatform alternative and augmentative communication tool, Procedia Computer Science 27(0): 197-206. http://dx. doi .org/10.1016/j. procs. 2014.02.023.

Carniel, A. and Berkenbrock, C. D. M. (2020). The use of augmentative and alternative communication tosupport people with intellectual disability, Revista Novas Tecnologias na Educação 18(1). http://dx. doi .org/10.22456/ 1679-1916.105920.

Caron, J. and Light, J. (2016). "social media has opened a world of 'open communication:'" experiences of adults with cerebral palsy who use augmentative and alternative communication and social media, Augmentative and Alternative Communication 32(1): 25-40. https://doi.org/10.3109/07434618.2015.1052887.

de Oliveira, A. I. A., Guimarães, L. d. S. and Sampaio Neto, N. C. (2013). Voicer in mobile platform to facilitate communication for the disabled, Proceedings of the Fifth International Conference on Management of Emergent Digital EcoSystems, pp. 320-325. http://dx.doi.org/10. $1145 / 2536146.2536197$.

El-Seoud, M. S. A., Karkar, A., Al Ja'am, J. M. and Karam, O. H. (2014). A pictorial mobile-based communication application for non-verbal people with autism, 2014 International Conference on Interactive Collaborative Learning (ICL), IEEE, pp. 529-534. http://dx.doi .org/ICL . 2014.7017828.

Flora, H. K. and Chande, S. V. (2014). A systematic study on agile software development methodologies and practices, International Journal of Computer Science and Information Technologies 5(3): 3626-3637. Disponível em http://www.ijcsit.com/docs/Volume\%205/ vol5issue03/ijcsit20140503214.pdf.

Fossett, B. and Mirenda, P. (2007). Augmentative and alternative communication, Handbook on developmental disabilities pp. 330-348.

Ganz, J. B., Hong, E. R. and Goodwyn, F. D. (2013). Effectiveness of the pecs phase iii app and choice between the app and traditional pecs among preschoolers with asd, Research in Autism Spectrum Disorders 7(8): 973-983. http://dx.doi.org/10.1016/j.rasd.2013.04.003.

Guisen, A., Sanz, C. and De Giusti, A. (2012). Ecca: Augmentative communication collaborative environment, 2012 International Conference on Collaboration Technologies and Systems (CTS), IEEE, pp. 282-285. http://dx. doi. org/10.1109/CTS. 2012.6261063.

Horner, R. H., Carr, E. G., Halle, J., McGee, G., Odom, S. and Wolery, M. (2005). The use of single-subject research to identify evidence-based practice in special education, Exceptional children 71(2): 165-179. http://dx.doi.org/ 10.1177/001440290507100203.

Huijbregts, T. and Wallace, J. R. (2015). Talkingtiles: Supporting personalization and customization in an aac app for individuals with aphasia, Proceedings of the 2015 International Conference on Interactive Tabletops \& Surfaces, pp. 63-72. http://dx.doi.org/10.1145/2817721. 2817723.
Luce, B. F. and Estabel, L. B. (2020). Letramento informacional e mídias sociais, Revista Brasileira de Pós-Graduação 16(35). https://doi. org/10.21713/rbpg.v16i35.1661.

Marques, C. V., Tolla, C. E., Motta, C., Vrabl, S., Lapolli, F., Ângelo, L. and Daflon, L. (2010). Avaliação de crianças deficientes visuais através de jogos neuropedagógicos, Revista Brasileira de Computação Aplicada 2(1): 28-40. http://dx.doi.org/10.5335/rbca.2013.721.

Thiessen, A. and Beukelman, D. (2019). Learning styles and motivations of individuals without prior exposure to augmentative and alternative communication, Topics in Language Disorders 39(1): 104-114. http://dx . doi .org/ 10.1097/TLD. 0000000000000167.

Wilson, C., Sitbon, L., Brereton, M., Johnson, D. and Koplick, S. (2016). 'put yourself in the picture' designing for futures with young adults with intellectual disability, Proceedings of the 28th Australian Conference on Computer-Human Interaction, pp. 271-281. http: $/ /$ dx.doi.org/10.1145/3010915.3010924. 